\title{
Near miss materno - entendendo e aplicando o conceito
}

\author{
Maternal near miss - understanding and applying the concept
}

\author{
Danielly Scaranello Santana, José Paulo Siqueira Guida, \\ Rodolfo Carvalho Pacagnella, José Guilherme Cecatti
}

Santana DS, Guida JPS, Pacagnella RC, Cecatti JG. Near miss materno - entendendo e aplicando o conceito / Maternal near miss understanding and applying the concept. Rev Med (São Paulo). 2018 mar.-abr.;97(2):187-94.

RESUMO: A morte materna é o evento mais raro e dramático, de uma cadeia de acontecimentos que pode acometer qualquer mulher durante o ciclo gravídico-puerperal. Esta cadeia de eventos inicia-se quando uma mulher apresenta alguma das diversas condições potencialmente ameaçadoras da vida (CPAV). Quando não adequadamente tratada, ela pode evoluir para condições ameaçadoras da vida, em que há disfunção ou falência orgânica e que se definem a partir de critérios clínicos, laboratoriais ou de manejo. Mulheres que sobrevivem a tais eventos graves serão classificadas como near miss materno. $\mathrm{O}$ conceito de near miss materno permite avaliação da qualidade da assistência obstétrica, representando uma ferramenta a ser utilizada na prática obstétrica rotineira, ao identificar mulheres que estejam sob maior risco de evolução para quadros graves, permitindo a rápida implementação de medidas de tratamento e suporte. O objetivo do presente artigo é revisar a construção histórica do conceito de near miss, apresentar os atuais parâmetros validados para a sua utilização e propor seu uso de forma rotineira, na prática clínica e no currículo dos cursos médicos e de especialização em Obstetrícia.

Descritores: Mortalidade materna; Morbidade; Saúde materna/ normas; Morte materna.
ABSTRACT: Maternal death is the most rare and dramatic event among a sequence of events that may affect any woman during pregnancy or up to 40 days post-partum. This sequence of events begins when a woman presents any of the potentially life-threatening conditions (PLTC). These conditions, when not properly treated, can evolve to life-threatening conditions when organ dysfunction of failure appear, which are defined using some clinical, laboratory, or management criteria. Women who survive such serious events will be classified as maternal near miss. The concept of maternal near miss allows the assessment of the quality of obstetric care, representing a tool to be used in the routine obstetric practice, for identifying women with higher risk of developing more severe conditions and allowing the rapid implementation of treatment and support measures. The objective of the current article is to review the historical construction of the concept of maternal near miss, to present the current validated parameters for use of this concept and to propose the routine use of maternal near miss in clinical practice and in the curriculum of medical schools and courses of specialization in Obstetrics.

Keywords: Maternal mortality; Morbidity; Maternal health/ standards; Maternal death.

Departamento de Tocoginecologia, Faculdade de Ciências Médicas da Universidade Estadual de Campinas, Campinas, SP, Brasil. ORCID/E-mail: Danielly S Santana, MD, PhD - http://orcid.org/0000-0001-8912-2626, dany.fmj@terra.com.br; Jose P Guida, MD, MSc - http://orcid.org/0000-0002-3648-6159, zepauloguida@gmail.com; Rodolfo C Pacagnella, MD, PhD - http://orcid.org/00000002-5739-0009, rodolfopacagnella@gmail.com; José G Cecatti, MD, PhD http://orcid.org/0000-0003-1285-8445, cecatti@unicamp.br Endereço para correspondência: José Guilherme Cecatti. Departmento de Obstetrícia e Ginecologia, Universidade Estadual de Campinas. Rua Alexander Fleming, 101. CEP: 13083-891. Campinas, SP, Brasil. E-mail: cecatti@unicamp.br. 


\section{INTRODUÇÃO}

A té a década de 80 , os índices de mortalidade materna e perinatal eram os principais indicadores da qualidade da assistência obstétrica, atingindo taxas próximas a 300 mortes maternas a cada 100.000 nascimentos em países de baixa renda, à época incluídos no chamado terceiro mundo. A mulher era considerada basicamente como um meio reprodutivo e os programas de saúde correlatos eram designados como de "saúde materno-infantil", fundamentados basicamente na saúde infantil, sendo por vezes o "M", de materno, esquecido. Em 1985 um artigo publicado por Rosenfield e Maine, relevando a importância da morte materna, iniciou uma série de discussões, reuniões, planejamentos e chamados que culminaram com o lançamento do Safe Motherhood Initiative (Iniciativa para a Maternidade Segura), na Conferência Internacional em Nairóbi, Quênia, em 1987, cujo objetivo era reduzir a mortalidade materna em $50 \%$ até o ano $2000^{1,2}$.

A partir de então, o programa, liderado pela Organização Mundial de Saúde (OMS), influenciou a inclusão do enfrentamento à mortalidade materna em programas de saúde de praticamente todos os países do mundo. Porém os resultados dessa iniciativa não se mostraram plenamente exitosos, já que muitos países ainda apresentavam razões de mortalidade maternas estáveis ou até mesmo mais elevadas em locais onde as informações passaram a ser melhor coletadas. $\mathrm{O}$ fracasso prático da Iniciativa para a Maternidade Segura foi responsável pela revisão dos conceitos e prioridades de formas de enfrentamento do problema.

Uma consequência da falência da Iniciativa para a Maternidade Segura foi a inclusão da Saúde Materna nas Metas de Desenvolvimento do Milênio pelas Nações Unidas no ano de 2000, propondo a redução em três quartos da taxa de mortalidade materna e acesso universal à saúde reprodutiva até 2015. Infelizmente, tais metas também não foram plenamente atingidas, o que levou as Nações Unidas a patrocinarem as Metas para o Desenvolvimento Sustentável, a serem atingidas até o ano de 2030. Neste escopo de objetivos, a Saúde Materna figura na terceira meta, objetivando-se uma redução das taxas de mortalidade materna para 70 a cada 100.000 nascidos vivos, além de propor acesso universal aos serviços de saúde sexual e reprodutiva, incluindo planejamento familiar e cobertura universal a serviços de saúde, medicamentos essenciais e vacinas, seguros, eficazes e de qualidade, até $2030^{3}$.

Entende-se atualmente que a morte materna seja o ponto final de uma cadeia de eventos já conhecidos, sobre a maioria dos quais podem ser realizadas intervenções. Além disso, apesar de ainda alarmante, o evento morte materna é raro, quando consideradas as taxas individualizadas de cada serviço, ao passo que os eventos que a precedem são muitas vezes mais frequentes. Desta forma, é importante compreender não somente a ocorrência da morte materna, mas também as condições que a precederam, com o objetivo de se entender quais são as intervenções possíveis para minorar este evento trágico.

O objetivo deste artigo é revisar a construção do conceito de near miss materno e a aplicação prática do mesmo em serviços de saúde.

\section{Morbidade Materna Grave e o conceito de near miss materno}

A morte materna é definida como qualquer morte ocorrida durante a gestação, parto ou até 42 dias de puerpério, por condições determinadas ou agravadas pela gravidez. Ela ainda é considerada o pior resultado obstétrico, correspondendo ao último evento de uma sequência que pode ocorrer na gestação. A maioria das gestações progredirá de uma forma saudável e fisiológica, finalizando-se com um parto sem intercorrências, gerando um concepto de peso e desenvolvimento adequado, com a plena recuperação da mulher ao fim do puerpério. Uma parcela das mulheres será, entretanto, acometida por complicações diversas, das menos graves às mais graves. Dentre as condições mais graves, algumas foram classificadas como condições potencialmente ameaçadoras da vida (CPAV). Estas condições associam-se a maior risco de progressão para gravidade e risco de vida, e foram estabelecidas pela Organização Mundial da Saúde (Quadro 1).

Quadro 1 - Condições potencialmente ameaçadoras da vida que podem acometer uma gestante ou puérpera

\begin{tabular}{|llll|}
\hline \multicolumn{4}{c}{ Condições potencialmente ameaçadoras da vida (CPAV) } \\
\hline Desordens hemorrágicas & Desordens hipertensivas & Outras desordens sistêmicas & Indicadores de manejo \\
\hline & & Endometrite & Transfusão sanguínea \\
Placenta prévia & Pré-eclâmpsia grave & Edema pulmonar & Acesso venoso central \\
Placenta acreta/increta/percreta & Eclâmpsia & Falência respiratória & Histerectomia \\
Gestação ectópica & Hipertensão grave & Convulsões & Admissão em UTI \\
Hemorragia pós-parto & Encefalopatia hipertensiva & Sepse & Internação prolongada (>7 dias) \\
Rotura uterina & HELLP síndrome & Trombocitopenia $<100.000$ & Intubação não anestésica \\
& & Crise tireotóxica & Intervenção cirúrgica \\
\hline
\end{tabular}

FONTE: Say L, Souza JP, Pattinson RC, WHO working group on Maternal Mortality and Morbidity classifications. Maternal near miss-towards a standard tool for monitoring quality of maternal health care. Best Pract Res Clin Obstet Gynecol. 2009;23:287-96. 
Por sorte ou por adequado tratamento, a maior parte das mulheres se recuperará da CPAV que apresentou, com pleno reestabelecimento, ou com algum grau de incapacidade, temporária ou permanente. Por outro lado, uma pequena fração destas mulheres evoluirá com complicações associadas à CPAV que lhe acometeu, complicações estas definidas pela deterioração clínica, a piora de parâmetros laboratoriais ou necessidade de medidas intensivas de cuidado, denotando algum grau de disfunção ou falência orgânica, com condições de fato ameaçadoras à vida e que se denominam atualmente near miss materno. A maior parte destas mulheres sobreviverá, com plena recuperação ou com algum grau de sequela, permanente ou temporária. Uma pequena fração, entretanto, terá o trágico desfecho da morte materna. A Figura 1 demonstra o contínuo de eventos que culmina com a morte materna.

\begin{tabular}{|c|c|c|c|c|}
\hline & & & & $\begin{array}{l}\text { Casos de near miss } \\
\text { materno }\end{array}$ \\
\hline & & & $\begin{array}{l}\text { Mulheres com condições } \\
\text { ameaçadoras da vida }\end{array}$ & \\
\hline & & $\begin{array}{l}\text { Mulheres com condições } \\
\text { potencialmente } \\
\text { ameaçadoras da vida }\end{array}$ & & Morte materna \\
\hline & $\begin{array}{l}\text { Mulheres com } \\
\text { complicações }\end{array}$ & & $\begin{array}{l}\text { Mulheres sem condições } \\
\text { ameaçadoras da vida }\end{array}$ & \\
\hline $\begin{array}{l}\text { Todas as mulheres } \\
\text { durante gestação, parto } \\
\text { e } 42 \text { dias pós-parto }\end{array}$ & & $\begin{array}{l}\text { Mulheres sem condições } \\
\text { potencialmente } \\
\text { ameaçadoras da vida }\end{array}$ & & \\
\hline & $\begin{array}{l}\text { Mulheres sem } \\
\text { complicações }\end{array}$ & & & \\
\hline
\end{tabular}

FONTE: Say L, Souza JP, Pattinson RC, WHO working group on Maternal Mortality and Morbidity classifications. Maternal near miss-towards a standard tool for monitoring quality of maternal health care. Best Pract Res Clin Obstet Gynecol. 2009;23:287-96.

Figura 1. A evolução da morbidade materna grave: da gestação não complicada à morte materna

Dessa forma, parece claro o entendimento de que os determinantes para as complicações muito graves associadas à gestação e para o óbito materno sejam basicamente os mesmos. Assim, o near miss materno, caracterizado por um conjunto de condições que caracterizam a disfunção ou falência orgânica e que antecede imediatamente o óbito, constitui um importante marcador de saúde e cuidados obstétricos. Seu nome é emprestado da indústria aeronáutica e se baseia em semelhante conceito para a avaliação dos acidentes ou "quase-acidentes" aeronáuticos não ocorri$\operatorname{dos}^{4,5,6}$. O near miss materno (NMM) é atualmente definido pela Organização Mundial de Saúde (OMS) como a mulher que quase morreu, mas sobreviveu a complicações graves durante a gestação, parto ou até 42 dias após o término da gestação. Seus critérios diagnósticos estão apresentados no Quadro $2^{6}$.

Quadro 2 - Critérios diagnósticos do near miss materno

\begin{tabular}{|c|c|c|}
\hline \multicolumn{3}{|c|}{ Near Miss Materno (NMM) } \\
\hline Critério clínico & Critério laboratorial & Critério de manejo \\
\hline $\begin{array}{l}\text { Cianose aguda } \\
\text { Gasping } \\
\text { Frequência respiratória }>40 \text { ou }<6 / \mathrm{min} \\
\text { Choque } \\
\text { Oligúria não responsiva a fluídos ou } \\
\text { diuréticos } \\
\text { Coagulopatia } \\
\text { Perda de consciência } \geq 12 \mathrm{~h} \\
\text { Perda de consciência e ausência de } \\
\text { pulso/ batimento cardíaco } \\
\text { Acidente vascular cerebral } \\
\text { Paralisia total } \\
\text { Icterícia na presença de pré-eclâmpsia }\end{array}$ & $\begin{array}{l}\text { Saturação de } \mathrm{O} 2<90 \% \text { por } \geq 60 \\
\text { minutos } \\
\mathrm{PaO}_{2} / \mathrm{FiO}_{2}<200 \mathrm{mmHg} \\
\text { Creatinina } \geq 300 \mu \mathrm{mol} / 1 \text { ou } \geq 3,5 \mathrm{mg} / \mathrm{dl} \\
\text { Bilirrubina }>100 \mu \mathrm{mol} / 1 \text { ou } 6,0 \mathrm{mg} / \mathrm{dl} \\
\mathrm{pH}<7,1 \\
\text { Lactato }>5 \\
\text { Trombocitopenia }(<50.000 \text { plaquetas }) \\
\text { Perda de consciência e presença de } \\
\text { gluconato ou cetoácidos na urina }\end{array}$ & $\begin{array}{l}\text { Uso contínuo de drogas vasoativas } \\
\text { Histerectomia por infecção ou hemorragia } \\
\text { Transfusão de } \geq 5 \text { unidades } \\
\text { Intubação e ventilação por } \geq 60 \text { minutos não } \\
\text { relacionada à anestesia } \\
\text { Diálise por falência renal aguda } \\
\text { Ressuscitação cardiopulmonar }\end{array}$ \\
\hline
\end{tabular}

FONTE: Say L, Souza JP, Pattinson RC, WHO working group on Maternal Mortality and Morbidity classifications. Maternal near miss-towards a standard tool for monitoring quality of maternal health care. Best Pract Res Clin Obstet Gynecol. 2009;23:287-96. 
O termo near miss materno foi citado pela primeira vez no final da década de 90, ainda sem definição dos critérios diagnósticos para a identificação dos casos, mas já surgindo como um útil marcador obstétrico. Porém o entendimento de que seu estudo sistemático seria de fato importante para uma mais fácil e completa compreensão das condições que contribuem para a morte materna é bem mais recente $^{4,7,8}$. Quando surgiu parecia ser particularmente útil em contextos onde a mortalidade materna era baixa, porém, na prática, tem se demonstrado útil nas mais diversas situações. Primeiro, porque as mulheres sobrevivem e assim podem relatar melhor os problemas que tiveram e as dificuldades que encontraram para receber o cuidado (9). Segundo, porque de forma diferente do que para a mortalidade materna, o conceito traz consigo o potencial positivo oposto ao de fracasso.

\section{Prevalência do near miss materno}

Os primeiros artigos que tentaram identificar critérios diagnósticos utilizaram-se de critérios clínicos ou de intervenções para identificar condições associadas a maior gravidade como admissão em UTI, hemorragia, pré-eclâmpsia grave, transfusão massiva de hemoderivados, histerectomia ${ }^{8,10}$. Porém somente no início dos anos 2000 que critérios baseados em disfunção orgânica, mais próximos aos atuais definidos, passaram a ser utilizados considerando uma sequência de eventos que levam desde um insulto clínico até a falência orgânica e morte ${ }^{11}$. Após esses primeiros artigos publicados, diversos estudos foram realizados visando identificar a prevalência dessa condição, porém sem utilização de critérios diagnósticos operacionalmente padronizados, as taxas de NMM variaram de 0,01 a $15 \%$. Estudos utilizando critérios clínicos identificaram uma prevalência variando de 0,6 a 14,9\%; os que utilizaram critérios de manejo identificaram uma prevalência de 0,04 a 4,54\%, enquanto os que utilizaram critérios baseados em disfunção orgânica identificaram prevalências variando de 0,14 a $0,92 \% \%^{5,12}$. Algumas revisões sistemáticas também já abordaram o tema dessa diversidade, basicamente todas recomendando que uma definição e critérios padronizados fossem utilizados para permitir a comparação de resultados entre diferentes contextos $^{5,12,13}$.

Assim em 2009 a OMS, após várias reuniões de um grupo internacional de especialistas no assunto, que constituiu uma força-tarefa para essa finalidade, padronizou o conceito de near miss materno, determinou seus critérios diagnósticos e indicadores de saúde utilizados para monitorar a qualidade dos resultados obstétricos nos casos de NMM e MM (Figura 2) (114 $^{6}$. A partir daí diversos outros estudos foram publicados, muitos deles utilizando esse novo conceito, mas em populações pequenas e serviços específicos. Dessa forma, alguns estudos multicêntricos ganham destaque porque, além de aplicarem o conceito de NMM definido pela OMS, utilizaram grandes populações na análise, identificando de forma homogênea a prevalência do NMM que variou de 9,37 a 12,3\%.

\begin{tabular}{|c|c|c|}
\hline Marcador & Cálculo & Significado \\
\hline $\begin{array}{l}\text { Potentially Life-Threatening } \\
\text { Conditions Ratio }\end{array}$ & $\begin{array}{l}\text { Número de casos de CPAV/ } 1000 \text { nascidos } \\
\text { vivos }\end{array}$ & \\
\hline Maternal Near Miss Ratio & $\begin{array}{l}\text { Número de casos de NMM/ } 1000 \text { nascidos } \\
\text { vivos }\end{array}$ & Estimativa da complexidade dos cuidados \\
\hline Severe Maternal Outcome Ratio & $\begin{array}{l}\text { Número de casos de NMM+MM/ } 1000 \\
\text { nascidos vivos }\end{array}$ & \\
\hline MNM:MD Ratio & $\begin{array}{l}\text { Proporção de casos de NMM para cada caso } \\
\text { que evoluiu para MM }\end{array}$ & \\
\hline Mortality index & Casos de MM/ Casos de NMM+MM & $\begin{array}{l}\text { Estimativa de desempenho, quanto mais } \\
\text { alto pior a qualidade da promoção de } \\
\text { cuidados obstétricos para os casos graves }\end{array}$ \\
\hline $\begin{array}{l}\text { Intrahospital Maternal Mortality } \\
\text { Ratio }\end{array}$ & $\begin{array}{l}\text { Número de mortes ocorridas nos primeiros } 7 \\
\text { dias pós-parto dentro do ambiente hospitalar } \\
\text { para } 100.000 \text { nascidos vivos }\end{array}$ & \\
\hline $\begin{array}{l}\text { Maternal Severity Score (MSS) } \\
\text { Maternal Severity Index }\end{array}$ & $\begin{array}{l}\text { Número de marcadores de gravidade para } \\
\text { cada caso de NMM+MM } \\
\text { Probabilidade de morte dentre os casos de } \\
\text { NMM+MM }\end{array}$ & $\begin{array}{l}\text { Monitoramento de performance de } \\
\text { cuidados fornecidos para a mulher com } \\
\text { complicações }\end{array}$ \\
\hline
\end{tabular}

FONTE: Say L, et al. Maternal near miss-towards a standard tool for monitoring quality of maternal health care. Best Pract Res Clin Obstet Gynecol 2009; 23:287-96. Souza JP, et al. Brazilian Network for Surveillance of Severe Maternal Morbidity. The WHO Maternal Near-Miss Approach and the Maternal Severity Index Model (MSI): Tools for Assessing the Management of Severe Maternal Morbidity. PLoS One. $2012 ; 8(8)$ :e44129.

Figura 2: Definição dos indicadores de cuidados obstétricos de acordo com OMS 
O primeiro estudo multicêntrico foi o da Rede Brasileira de Vigilância de Morbidade Materna Grave, realizado em 27 unidades obstétricas de referência em diferentes regiões do Brasil, de julho de 2009 a junho de 2010, com objetivo de realizar uma vigilância prospectiva dos casos de CPAV, NMM e MM. Este estudo identificou 9.555 mulheres que apresentaram algum tipo de complicação grave na gestação, com uma prevalência de near miss materno de 9,37 por mil nascidos vivos ${ }^{15}$. Outro estudo multicêntrico com objetivo de avaliar a morbidade e mortalidade materna e perinatal foi o WHO Multicountry Survey on Maternal and Neonatal Health, desenvolvido de maio de 2010 a dezembro de 2011 em 359 instituições de 29 países, incluindo 314.623 mulheres, das quais 23.015 apresentaram algum tipo de complicação na gestação, com uma prevalência de NMM de 9,9 por mil nascidos vivos $^{16}$. Mais recentemente um estudo da Organização Pan Americana de Saúde (OPAS), desenvolvido em 20 instituições de 12 países da América Latina de março a junho de 2013, identificou em 3.196 mulheres incluídas no estudo, uma prevalência de NMM de 12,3 por mil nascidos $\operatorname{vivos}^{17}$.

\section{O near miss materno como ferramenta de vigilância de morbidade materna e qualidade de atenção à saúde materna}

Se a complicação com maior potencial de evoluir mais gravemente e para a morte for detectada a tempo e medidas adequadas forem tomadas pelos serviços e profissionais de saúde, há a possibilidade de se alterar a evolução do caso e a mulher pode sobreviver. Ao mesmo tempo, a chegada de uma mulher aos serviços de referência em condições de gravidade que podem levá-la à morte pode representar a desorganização dos serviços de saúde. Nesse sentido tais características podem fundamentar a concretização de um sistema real de vigilância de morbidade materna grave, que permita intervenções rápidas nos mais diversos níveis do sistema ${ }^{18}$.

Desde sua padronização oficial pela OMS em 2009, os critérios diagnósticos do near miss têm como propósito prático ser utilizado como uma ferramenta de monitoramento da qualidade da atenção à saúde materna ${ }^{6,19}$. A validação desses critérios foi realizada inicialmente de forma retrospectiva com a análise dos prontuários de 673 mulheres admitidas em UTI no período de outubro de 2002 a setembro de 2007, comparando-se os critérios diagnósticos de near miss com o escore SOFA (Sequencial Organ Failure Assesment), padrão-ouro de quantificação de falência orgânica, desenvolvido com o objetivo de definir critérios que mais provavelmente demonstrassem a totalidade das disfunções e falências orgânicas, e que deram suporte para os critérios desenvolvido pela OMS. Concluiuse que os critérios do near miss materno determinados pela OMS foram capazes de identificar todos os casos de morte e quase todos os casos de falência orgânica, apresentando uma sensibilidade de $99,2 \%$ e especificidade de $86,0 \%$ na identificação de falência orgânica ${ }^{20,21,22}$. A Rede Brasileira de Vigilância da Morbidade Materna Grave, através de análise prospectiva dos casos de morbidade materna grave, também permitiu a validação desses critérios com uma sensibilidade de $100 \%$ e especificidade de $92 \%{ }^{23,24}$. Os resultados desse mesmo estudo acabam de fornecer outra validação prospectiva do conceito e critérios da OMS para near miss materno, demonstrando outra vez excelente desempenho ${ }^{25}$.

O uso rotineiro dos critérios diagnósticos na identificação dos casos de maior gravidade materna por comitês hospitalares ou grupos semelhantes é ferramenta útil na vigilância da ocorrência da morbidade materna grave e da qualidade de cuidados obstétricos, porém não são suficientes. Juntamente com o desenvolvimento dos critérios diagnósticos, a OMS desenvolveu indicadores de saúde com o objetivo de facilitar a comparação da situação da morbidade materna e secundariamente a qualidade dos cuidados obstétricos ao longo do tempo. São eles: Maternal Near Miss Ratio, Severe Maternal Outcome, Maternal Near Miss: Maternal Mortality Ratio e Mortality Index ${ }^{6}$. Porém esses indicadores não se mostraram suficientes e novos indicadores foram sendo desenvolvidos de acordo com a necessidade de se prever e avaliar a morbidade e mortalidade da população obstétrica, de comparar serviços obstétricos e até mesmo diferentes momentos de um serviço que tenha realizado alguma intervenção. Surgiu assim o Maternal Severity Index, ferramenta que avalia o desempenho dos serviços de saúde em mulheres que apresentaram complicações graves e prevê a mortalidade nessa população, e o Maternal Severity Score (Figura 2) $)^{25,26}$. Mais recentemente utilizou-se um marcador denominado Standardized Mortality Ratio (SMR) que corresponde à razão entre mortalidade observada na população e mortalidade esperada por predição de mortalidade com base na gravidade do caso. Esse marcador é capaz de avaliar cada centro de saúde especificamente levando em consideração não somente situações associadas ao serviço de saúde como estrutura, gerenciamento e atrasos, mas também situações associadas ao acesso ao serviço de saúde e comunidade ${ }^{26}$.

Operacionalmente, o near miss pode ser utilizado a partir de duas perspectivas: retrospectiva ou prospectiva. Quando se avalia a qualidade de assistência de certo serviço, a identificação dos casos de near miss e morte materna permite identificar as falhas de processo ou atrasos que levaram à instalação da condição, bem como as medidas que permitiram que o desfecho morte fosse evitado. Quando utilizado a partir de uma perspectiva prospectiva, a identificação precoce de mulheres com CPAV ou com critérios de near miss permite que tais mulheres sejam rápida e adequadamente tratadas, evitando que o desfecho morte se instale.

A vigilância da morbidade materna mostra-se 
diretamente relacionada à qualidade de atendimento dos serviços de saúde. A identificação dos casos de morbidade materna grave juntamente com o cálculo dos indicadores de saúde permite uma adequada vigilância em saúde, e se essa vigilância for realizada rotineiramente, pontos fracos de cada serviço poderão ser identificados. O reconhecimento desses pontos permitirá a adequação de cada serviço às necessidades da população a ser atendida, melhorando assim a qualidade de atendimento.

\section{O conceito de near miss materno em inquéritos populacionais}

Como um instrumento para ser utilizado em tempo real, tentando identificar as complicações à medida que elas apareçam, os critérios de near miss materno dependem fundamentalmente de informações clínicas e laboratoriais que precisam ser conferidas diariamente. Dessa forma surgiu a discussão de sua utilização em inquéritos populacionais onde as próprias mulheres seriam a fonte de informação dessa ocorrência. Uma revisão sistemática veio então mudar esse pensamento ao identificar que era possível utilizar o conceito de near miss materno em inquéritos populacionais para que as próprias mulheres fornecessem a informação sobre a morbidade que tinham vivenciado no passado, desde que se utilizassem formulários especialmente desenhados e validados para isso ${ }^{27}$. Também foi demonstrada a possibilidade de utilização de bancos de dados com informações sobre a atenção ao nascimento como uma medida indireta da ocorrência de morbidade materna grave ${ }^{28}$.

Com esse propósito, desenvolveu-se e validouse um questionário com o objetivo de identificar, entre as mulheres entrevistadas em inquéritos populacionais, as que tivessem tido complicações maternas em seus partos passados, que pudessem ser classificadas como near miss materno. Esse questionário utilizava perguntas padronizadas sobre a ocorrência de determinadas situações clínicas mais comumente associadas à gravidade da complicação e também sobre procedimentos mais comumente utilizados para o manejo destas situações ${ }^{29}$. Este questionário foi incluído na última PNDS (Pesquisa Nacional de Demografia e Saúde) do Brasil e os resultados revelaram uma prevalência de cerca de $23 \%$ de gestações com complicações e uma razão ajustada de near miss materno de 21,1 casos por 1.000 nascidos vivos, quando se considerou o conjunto de indicadores constituído por eclâmpsia, histerectomia, admissão em UTI, transfusão sanguínea ou qualquer associação destes. Este conjunto demonstrou a melhor capacidade preditiva para identificação dos óbitos maternos (30). Da mesma forma, demonstrou uma ocorrência proporcionalmente maior de near miss materno entre as gestações com complicações terminadas em aborto do que as terminadas em parto $^{31}$.

Desta forma, fica claro que o conceito de near miss materno, e as ferramentas dele derivadas, podem ser utilizadas em pesquisas e inquéritos populacionais, bem como podem ser utilizados como ferramenta para avaliação de qualidade de determinado serviço de saúde, para avaliar o impacto de determinadas medidas e alterações nos processos de trabalho.

\section{O conceito do Near Miss Materno no dia-a-dia}

Além de ser uma ferramenta plenamente validada para o uso em inquéritos populacionais e de "audit and feedback" de serviços de saúde, o near miss materno pode ser utilizado no dia-a-dia da assistência obstétrica. É importante que o conceito seja disseminado entre médicos obstetras, enfermeiros, residentes de ginecologia e obstetrícia e estudantes, de forma a permitir a rápida identificação dos casos com potencial evolução para gravidade.

Os Quadros 1 e 2 mostram os critérios de potencial gravidade e aqueles nas quais a gravidade já se encontra estabelecida. A identificação das condições potencialmente ameaçadoras da vida (CPAV) é, em grande parte, possível a partir de uma avaliação clínica detalhada, não demandando, na maior parte das vezes, grandes recursos laboratoriais ou de imagem. A mulher acometida por estas condições deve ser referenciada para serviço que disponha de profissionais habilitados e estrutura adequada. Em já estando em tais serviços, é importante que esta mulher seja priorizada no seu atendimento e receba atenção e cuidados, de manejo e suporte, adequados.

Assim, além de um conceito teórico, o near miss é também um conceito operacional, com bases já cristalizadas e com facilidade em sua aplicação. A disseminação do conceito tem o potencial de modificar a atual assistência obstétrica e é fundamental na formação dos futuros profissionais e atualização daqueles que já atuam nestes cenários.

\section{CONCLUSÃO}

A estrutura conceitual do near miss materno foi desenvolvida ao longo dos últimos dez anos, como parte de um esforço de um grupo internacional de especialistas da Organização Mundial da Saúde. Ferramentas que derivaram deste conceito, como já discutido, mostraram-se válidas para a identificação dos casos graves e aplicáveis nos mais diversos locais.

É importante que o médico entenda a ferramenta não apenas como um instrumento de avaliação de qualidade de assistência, mas sim como uma maneira de, na prática diária, identificar os casos potencialmente graves, bem como aquelas já acometidos por complicações, e poder intervir, de forma rápida e eficaz, para evitar o agravamento ou até o óbito materno. Os critérios de near miss já estão claramente estabelecidos, e o seu reconhecimento não 
Santana DS, et al. Near miss materno - entendendo e aplicando o conceito.

demanda, na maior parte das vezes, grande estrutura laboratorial.

Assim, identificar as mulheres sob risco permitirá a rápida intervenção e ajudará em seu pleno reestabelecimento,

\section{REFERÊNCIAS}

1. Rosenfield A, Maine D. Maternal mortality - a neglected tragedy. Where is the M in MCH? Lancet. 1985;2(8446):835. https://doi.org/10.1016/S0140-6736(85)90188-6.

2. Starrs AM. Safe motherhood initiative: 20 years and counting. Lancet. 2006;368(9542):1130-1132. DOI: 10.1016/ S0140-6736(06)69385-9.

3. United Nations Development Programme - UNDP. The Millennium Development Goals. Eight goals for 2015. UNDP 2000 [cited 2012 Sept 03]. Available from: http://www.undp. org/content/undp/en/home/mdgoverview.html.

4. Pattinson RC, Hall M. Near misses: a useful adjunct to maternal death enquiries. Br Med Bull. 2003;67:231-43.

5. Say L, Pattinson RC, Gülmezoglu AM. WHO systematic review of maternal morbidity and mortality: the prevalence of severe acute maternal morbidity (near miss). Reprod Health. 2004;1(1):3. doi: 10.1186/1742-4755-1-3.

6. Say L, Souza JP, Pattinson RC; WHO working group on Maternal Mortality and Morbidity classifications. Maternal near miss-towards a standard tool for monitoring quality of maternal health care. Best Pract Res Clin Obstet Gynaecol. 2009;23(3):287-96. doi: 10.1016/j.bpobgyn.2009.01.007.

7. Stones W, Lim W, Al-Azzawi F, Kelly M. An investigation of maternal morbidity with identification of life threatening 'near miss' episodes. Health Trends. 1991;23(1):13-15.

8. Bewley S, Creighton SB. 'Near-miss' obstetric enquiry. J Obstet Gynecol. 1997;17(1):26-9. doi: 10.1080/01443619750114031.

9. Souza JP, Cecatti JG, Parpinelli MA, Krupa F, Osis MJ. An emerging "maternal near-miss syndrome": narratives of women who almost died during pregnancy and childbirth. Birth. 2009;36(2):149-58. doi: 10.1111/j.1523$-536 X .2009 .00313 . x$.

10. Mantel GD, Buchmann E, Rees H, Pattinson RC. Severe acute maternal morbidity: a pilot study of a definition for a near-miss. Br J Obstet Gynaecol. 1998;105(9):985-90.

11. Waterstone M, Bewley S, Wolfe C. Incidence and predictors of severe obstetric morbidity: case-control study. BMJ. 2001;322(7294):1089-93. doi: https://doi.org/10.1136/ bmj.322.7294.1089.

12. Tunçalp O, Hindin MJ, Souza JP, Chou D, Say L. The prevalence of maternal near miss: a systematic review. BJOG. 2012;119(6):653-61. doi: 10.1111/j.1471-0528.2012.03294.x.

13. Souza JP, Cecatti JG, Parpinelli MA, De Sousa MH, Serruya SJ. Revisão sistemática sobre morbidade materna near miss. Cad Saude Publica. 2006;22(2):255-64. DOI: /S0102$-311 X 2006000200003$. motivo pelo qual o conceito deve ser reconhecido por médicos, residentes e estudantes, o que poderá alterar, de forma substancial, o atual cuidado provido na assistência obstétrica.

14. Pattinson R, Say L, Souza JP, Broek N, Rooney C. WHO Working Group on Maternal Mortality and Morbidity Classifications. WHO maternal death and near-miss classifications. Bull World Health Organ. 2009;87(10):734. doi: 10.2471/ BLT.09.071001.

15. Cecatti JG, Costa ML, Haddad SM, Parpinelli MA, Souza JP, Sousa MH, Surita FG, Pinto E Silva JL, Pacagnella RC, Passini RJ; Brazilian Network for Surveillance of Severe Maternal Morbidity study group. Network for Surveillance of Severe Maternal Morbidity: a powerful national collaboration generating data on maternal health outcomes and care. BJOG. 2016;123(6):946-53. doi: 10.1111/1471-0528.13614.

16. Souza JP, Gülmezoglu AM, Vogel J, Carroli G, Lumbiganon P, Qureshi Z, et al. Moving beyond essential interventions for reducing of maternal mortality the WHO Multicountry Survey on Maternal and Newborn Health: a cross-sectional study. Lancet. 2013;381(9879):1747-55. doi: 10.1016/ S0140-6736(13)60686-8.

17. De Mucio B, Abalos E, Cuesta C, Carroli G, Serruya S, Giordano D, Martinez G, Sosa CG, Souza JP; the Latin American Near Miss Group (LANe-MG). Maternal near miss and predictive ability of potentially life-threatening conditions at selected maternity hospitals in Latin America. Reprod Health. 2016;13(1):134. https://doi.org/10.1186/ s12978-016-0250-9.

18. Sousa MH, Cecatti JG, Hardy EE, Serruya SJ. Severe maternal morbidity (near miss) as a sentinel event of maternal death. An attempt to use routine data for surveillance. Reprod Health 2008; 28:5-6.

19. World Health Organization (WHO). Evaluating the quality of care for severe pregnancy complications: the WHO near-miss approach for maternal health. Geneva; 2011 [cited 2012 Sept. 03]. Available from: http://whqlibdoc.who.int/ publications/2011/9789241502221_eng.pdf.

20. Oliveira-Neto AF, Parpinelli MA, Cecatti JG, Souza JP, Sousa MH. Sequential Organ Failure Assessment score for evaluating organ failure and outcome of severe maternal morbidity in obstetric intensive care. Scientific World J. 2012;1:1-9. doi: 10.1100/2012/172145

21. Vincent JL, De Mendonça A, Cantraine F, Moreno R, Takala J, Suter PM, Sprung CL, Colardyn F, Blecher S. Use of the SOFA score to assess the incidence of organ dysfunction/ failure in intensive care units: results of a multicentric, prospective study. Working group on "sepsis-related problems" of the European Society of Intensive Care Medicine. Crit Care Med. 1998;26:1783-00.

22. Cecatti JG, Souza JP, Oliveira Neto AF, Parpinelli MA, Sousa MH, Say L, Pattinson RC. Pre-validation of the $\mathrm{WHO}$ organ dysfunction based criteria for identification of 
Santana DS, et al. Near miss materno - entendendo e aplicando o conceito.

maternal near miss. Reprod Health. 2011;8:22. https://doi. org/10.1186/1742-4755-8-22.

23. Cecatti JG, Souza JP, Parpinelli MA, Haddad SM, Camargo RS, et al. Brazilian network for the surveillance of maternal potentially life threatening morbidity and maternal nearmiss and a multidimensional evaluation of their long term consequences. Reprod Health. 2009;6:15. doi: 10.1186/17424755-6-15.

24. Haddad SM, Cecatti JG, Parpinelli MA, Souza JP, Costa ML, Surita FG, et al. From planning to practice: building the national network for the Surveillance of Severe Maternal Morbidity. BMC Public Health. 2011;11:283. https://oi. org/10.1186/1471-2458-11-283.

25. Souza JP, Cecatti JG, Haddad SM, Parpinelli MA, Costa ML, Katz L, Say L, on behalf of the Brazilian Network for Surveillance of Severe Maternal Morbidity Group. The WHO maternal near miss approach and the Maternal Severity Index Model (MSI): tools for assessing the management of severe maternal morbidity. Plos ONE. 2012;7(8). 7(8):e44129. https://doi.org/10.1371/journal.pone.0044129

26. Haddad SM, Cecatti JG, Souza JP, Sousa MH, Parpinelli MA, Costa ML, et al. Applying the maternal near miss approach for the evaluation of quality of obstetric care: a worked example from a Multicentre Surveillance Study. Biomed Res Int. 2014;2014:989815. http://dx.doi.org/10.1155/2014/989815

27. Souza JP, Parpinelli MA, Amaral E, Cecatti JG. Population surveys using validated questionnaires provided useful information on the prevalence of maternal morbidities. J Clin Epidemiol. 2008;61:169-176. https://doi.org/10.1016/j. jclinepi.2007.04.009.

28. Souza JP, Cecatti JG, Faundes A, Morais SS, et al. World Health Organization 2005 Global Survey on Maternal and Perinatal Health Research Group. Maternal near miss and maternal death in the World Health Organization's 2005 global survey on maternal and perinatal health. Bull World Health Organ. 2010;88,(2):113-9. doi: 10.2471/ BLT.08.057828.

29. Souza JP, Cecatti JG, Pacagnella RC, Giavarotti TM, Parpinelli MA, Camargo RS, Sousa MH. Development and validation of a questionnaire to identify severe maternal morbidity in epidemiological surveys. Reprod Health. 2010;7:16. https://doi.org/10.1186/1742-4755-7-16.

30. Souza JP, Cecatti JG, Parpinelli MA, Sousa MH, Lago TG, Pacagnella RC, Camargo RS. Maternal morbidity and near miss in the community: findings from the 2006 Brazilian demographic health survey. BJOG. 2010;117(13):1586-92. doi: 10.1111/j.1471-0528.2010.02746.x.

31. Camargo RS, Santana DS, Cecatti JG, Pacagnella RC, Tedesco RP, Melo EF Jr, Sousa MH. Severe maternal morbidity and factors associated with the occurrence of abortion in Brazil. Int J Gynaecol Obstet. 2011;112(2):88-92. Doi: 10.1016/j.ijgo.2010.08.013. 